\title{
High-resolution, non-scanning FTS for remote sensing
}

\author{
K. Døhlen ${ }^{1}$ and A. Cañas
}

The Blackett Laboratory, Imperial College of Science and Technology, London SW7, UK

\section{Introduction}

We present the first results from a portable spectrometer for the visible and very near infrared based upon the principle of heterodyned holographic Fourier transform spectroscopy (HHS) (Dohi and Suzuki 1971, Dohlen 1994). The instrument uses a Michelson interferometer where one of the mirrors is replaced with a grating. This produces a spatially located, frequency-shifted interferogram which is read out by an all-reflective relay lens and a photo-diode array and processed on a portable PC. A battery pack ensures an autonomy of about 7 hours. Instrumental assets include high optical throughput, variable resolving power, and no moving parts.

We have successfully used the instrument in two different remote sensing applications: detection of vegetation reflectance and atmospheric absorption.

\section{The vegetation red edge}

Green vegetation absorbs most of the visible ratiation, but in the near infrared it is highly reflective. A sharp spectral edge (the "red edge") separates the two regions, its shape and position are diagnostic of the health and state of growth for the plant (Horler et. al. 1983).

Since diagnostic features of the red edge are brought out by differentiation, good noise performance is necessary. Usually, field-based reflectance measurements require extensive filtering or curve-fitting in order to reach acceptable noise levels. Using band-limited spectra of low resolution $(10 \mathrm{~nm})$ we have obtained sufficiently good noise performance $(S N R=1000)$ to allow numerical differentiation without further signal processing. In Figure 1 the solid line shows the measured red-edge spectrum and the dotted line its derivative. Two prominent features seen in the derivative spectrum are, according to Horler et. al. (1983), due to the chlorophyll absorption edge (the plateau at $710 \mathrm{~nm}$ ) and scattering within the canopy (the peak at $725 \mathrm{~nm}$ ).

\footnotetext{
${ }^{1}$ Presently at: Laboratoire d'Optique, Observatoire de Marseille, 2 Place le Verrier, 13248 Marseille Cedex, France
} 


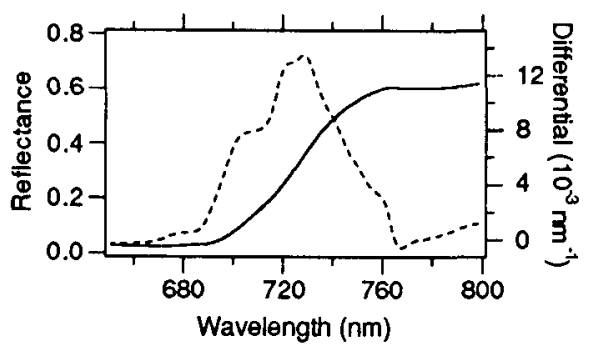

Figure 1. The red edge of a green-plant reflectance spectrum (solid line, left axis) and its first derivative (dotted line, right axis).

\section{Atmospheric $\mathrm{NO}_{2}$ absorption}

$\mathrm{NO}_{2}$ is an atmospheric pollutant whose concentration monitoring is of interest in remote sensing. We have studied a $2 \mathrm{~nm}$-wide $\mathrm{NO}_{2}$ absorption band centred at $489 \mathrm{~nm}$ at a spectral resolution of $0.2 \mathrm{~nm}$. Two blue-sky spectra were measured, one towards the London horizon, the other towards the Zenith. Ratioing the two, we have detected a signal corresponding to the difference between absorption along the two paths. Transmittance through a path length $L$ of a gas at partial pressure $p$ is given by the Lambert-Beer law: $T=\exp (-p L \alpha)$, where $\alpha$ is the absorption coefficient. Taking the natural logarithm of our spectral ratio and using a pressure-path-length product of $0.015 \mathrm{~cm}$ atm, we obtain a good fit with a published $\mathrm{NO}_{2}$ absorption-coefficient spectrum (Woods and Jolliffe 1978), see Figure 2. Assuming the path length to equal the meteorological range of a "standard clear atmosphere", i.e. $23.5 \mathrm{~km}$, the partial pressure of $\mathrm{NO}_{2}$ over London was hence estimated to $6 \mathrm{ppb}$ (parts per billion).

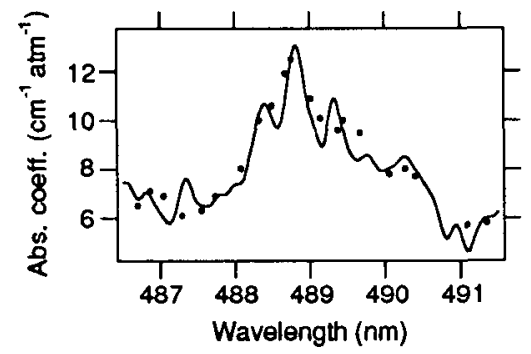

Figure 2. Comparison of our measured $\mathrm{NO}_{2}$ absorption coefficient (solid line) and that published by Woods and Jolliffe (1978) (points).

\section{References}

Dohi, T. and Suzuki, T. 1971, Appl.Optics, 10, 1137

Døhlen, K. 1994, Ph.D. Thesis, University of London

Horler, D., Dockray, M., and Barber, J. 1983, Int. J. Remote Sens., 4, 273

Woods, P. and Jolliffe, B. 1978, Optics and Laser Techn., Feb. 1978, p. 25 